\title{
Low-Temperature Series for the Square Lattice Potts Model by the Improved Finite-Lattice Method
}

\author{
H. ARISUE \\ Osaka Prefectural College of Technology \\ Saiwai-cho, Neyagawa, Osaka 572, Japan \\ K. TABATA \\ Osaka Institute of Technology \\ Junior College \\ Ohmiya, Asahi-ku, Osaka 535, Japan
}

September 5, 2018

\begin{abstract}
The low-temperature series are calculated for the free energy, magnetization and susceptibility in the $Q$-state Potts model on the square lattice, using the improved algorithm of the finite lattice method. The series are obtained to the order of $z^{41}$ for each of $Q=5-50$, and the result of their Padé type analysis is compared with those of the large- $Q$ expansion and the Monte Carlo simulations.
\end{abstract}




\section{Introduction}

The finite-lattice method is a very efficient technique to generate the expansion series in statistical models [1, 2] and in lattice gauge theory [3]. In this method the expansion series for the free energy density in the infinite-volume limit is given by an appropriate linear combination of the free energies on finite-size lattices. The partition function of the finite-size lattices, from which the free energy of the lattices is obtained, can be calculated using the transfer matrix formalism 㘬. This method avoids the problem involved in the graphical method, in which it is rather difficult to give the algorithm for listing all the diagrams that contribute to the relevant order of the series. The maximum order of the expansion series is determined by the maximum size of the lattices whose partition function can be calculated exactly to the order.

In the case of the model whose spin-variable at each site takes many discrete values, however, one can only calculate the partition function of the finite-size lattices whose sizes are relatively small and it is difficult to obtain the series to such high order as in the Ising model, in which the spin variable takes only two values. Recently one of the authors [5] proposed an improved algorithm of the finite-lattice method, which enables one to obtain higher order terms than the standard finite-lattice method in the spin systems whose spin variables take a large or infinite number of discrete values. It was applied to the low-temperature expansion for the free energy of the solid-on-solid (SOS) model and it enabled him to extend the series to the order of $u^{23}$ [5] from the order of $u^{12}$ that was obtained using the standard finite-lattice method [6].

In this paper we apply this improved finite lattice method to the calculation of the low-temperature series for the free energy, magnetization and susceptibility in the $Q$-state Potts model on the square lattice. This system has the first order phase transition for $Q \geq 5$ [7]. The values of the free energy, latent heat, and the gap of the specific heat and magnetization at the critical point are known exactly [8], while the exact values of the specific heat itself and the magnetic susceptibility at the critical point are not known yet. We calculate the series to order $z^{41}$ for each of $Q=5-50$. We can extend the series from the ones calculated previously by Bhanot et al [9] and Briggs et al [11] using the standard finite-lattice method, to substantially higher orders for large values of $Q \leq 10$, and our series for $11 \leq Q \leq 50$ have the order that is about 3 times higher than the ones given by Kihara et al for general $Q$ and zero field [12] or by Straley and Fisher [13] for general $Q$ 
and general field. We analyze the obtained series using the inhomogeneous differential approximants and their integration. It serves to check the validity of the series obtained and of the method of the analysis used for the quantities whose values are known exactly. On the other hand, it makes a new prediction for the quantities whose exact values are not known.

In section 2, we describe the improved algorithm of the finite-lattice method for the low-temperature expansion of the $Q$-state Potts model on the square lattice. The series obtained by the improved algorithm of the finite-lattice method are given in section 3. We present the analysis of the series in section 4. The result of our analysis is compared with those of the large- $Q$ expansion and the Monte Carlo simulations. Our results are summarized in section 5 .

\section{Algorithm}

Here we give the improved algorithm of the finite-lattice method for the low-temperature expansion of the $Q$-state Potts model on the square lattice, which enables us to obtain longer series than the standard finite-lattice method. Let us consider the two-dimensional $L_{x} \times L_{y}$ rectangular lattice $\Lambda_{0}$. The free energy density in the infinite-volume limit is given by

$$
f=-\lim _{L_{x}, L_{y} \rightarrow \infty} \frac{1}{L_{x} L_{y}} \ln \left[Z\left(\Lambda_{0} ; Q\right)\right],
$$

with

$$
Z\left(\Lambda_{0} ; Q\right)=\sum_{\left\{0 \leq s_{i} \leq Q-1\right\}} \exp \left[-\beta \sum_{<i, j>}\left(1-\delta_{s_{i}, s_{j}}\right)-h \sum_{i}\left(1-\delta_{s_{i}, 0}\right)\right]
$$

where the integer variable $s_{i}$ at each site $i$ of $\Lambda_{0}$ is restricted to $0 \leq s_{i} \leq$ $Q-1$. The low-temperature series is calculated with respect to the expansion parameter $z=\exp (-\beta)$. We take the boundary condition that all the variables outside $\Lambda_{0}$ are fixed to be zero.

We consider the set $\{\Lambda\}$ of all two-dimensional rectangular sub-lattices of $\Lambda_{0}$. The sub-lattice $\Lambda$ is denoted by its size $l_{x} \times l_{y}$ and its position in $\Lambda_{0}$. We define a set of integers $\xi_{q}$ that consist of 0 and $(q-1)$ integers among the set of integers $\{1,2, \ldots, Q-1\}$. We define the $H$ of $\Lambda$ and $\xi_{q}$ as

$$
H\left(\Lambda ; \xi_{q}\right)=-\ln \left[Z\left(\Lambda ; \xi_{q}\right)\right]
$$


In the calculation of the partition function $Z\left(\Lambda ; \xi_{q}\right)$, the variable $s_{i}$ at each site $i$ inside $\Lambda$ is restricted to be one of the elements of $\xi_{q}$, and all the variables outside $\Lambda$ are fixed to be zero. We define $W$ of $\Lambda$ and $\xi_{q}$ recursively as

$$
W\left(\Lambda ; \xi_{q}\right)=H\left(\Lambda ; \xi_{q}\right)-\sum_{\substack{\Lambda^{\prime} \subseteq \Lambda, \xi_{q^{\prime}} \subseteq \xi_{q}}} W\left(\Lambda^{\prime} ; \xi_{q^{\prime}}\right) .
$$

Here the prime in the summation $\sum^{\prime}$ implies that the $W\left(\Lambda^{\prime}=\Lambda ; \xi_{q^{\prime}}=\xi_{q}\right)$ should be excluded in taking the summation. We note that the $H\left(\Lambda ; \xi_{q}\right)$ and $W\left(\Lambda ; \xi_{q}\right)$ depend on the size of $\Lambda$ but not on its position and depend on the value of $q$ but not on the details of the elements of the $\xi_{q}$. So we denote them as $H\left(l_{x} \times l_{y} ; q\right)$ and $W\left(l_{x} \times l_{y} ; q\right)$, then we can rewrite Eq. (坐) as

$$
\begin{aligned}
& W\left(l_{x} \times l_{y} ; q\right)=H\left(l_{x} \times l_{y} ; q\right) \\
& -\sum_{\substack{l_{x}^{\prime} \leq l_{x}, l_{y}^{\prime} \leq l_{y}, 2 \leq q^{\prime} \leq q}}^{\prime}\left(l_{x}-l_{x}^{\prime}+1\right)\left(l_{y}-l_{y}^{\prime}+1\right)\left(\begin{array}{c}
q-1 \\
q^{\prime}-1
\end{array}\right) W\left(l_{x}^{\prime} \times l_{y}^{\prime} ; q^{\prime}\right) .
\end{aligned}
$$

We know

$$
\begin{gathered}
H\left(L_{x} \times L_{y} ; Q\right)=\sum_{\substack{l_{x} \leq L_{x}, l_{y} \leq L_{y}, q \leq Q}}\left(L_{x}-l_{x}+1\right)\left(L_{y}-l_{y}+1\right)\left(\begin{array}{c}
Q-1 \\
q-1
\end{array}\right) \\
\times W\left(l_{x} \times l_{y} ; q\right)
\end{gathered}
$$

Taking the infinite-volume limit we obtain

$$
\begin{aligned}
f & =\lim _{L_{x}, L_{y} \rightarrow \infty} \frac{1}{L_{x} L_{y}} H\left(L_{x} \times L_{y} ; Q\right) \\
& =\sum_{l_{x}, l_{y}, q \leq Q}\left(\begin{array}{c}
Q-1 \\
q-1
\end{array}\right) W\left(l_{x} \times l_{y} ; q\right) .
\end{aligned}
$$

The magnetization $M$ and susceptibility $\chi$ are given from this free energy density, by

$$
M=1-\frac{Q}{Q-1} \frac{\partial f}{\partial h}
$$

and

$$
\chi=\frac{\partial^{2} f}{\partial h^{2}} .
$$


In the practical calculation we introduce the variable $x \equiv \exp (h)-1$ [14], then

$$
\left.\frac{\partial}{\partial h}\right|_{h=0}=\left.\frac{\partial}{\partial x}\right|_{x=0}
$$

and

$$
\left.\frac{\partial^{2}}{\partial h^{2}}\right|_{h=0}=\left.\left(\frac{\partial^{2}}{\partial x^{2}}+\frac{\partial}{\partial x}\right)\right|_{h=0}
$$

and it is enough to evaluate the expansion series for the free energy density to the second order in $x$ in order to obtain the zero-field magnetization and susceptibility.

In the standard cluster expansion of the free energy [15, 16] for this model, a cluster is composed of polymers and each of the polymers consists of the excited sites that are connected by nearest-neighbor bonds. An integer value $s_{i} \in\{1,2, \ldots, Q-1\}$ is put to each excited site $i$ of the polymer. For each cluster we can define the subset of the set $\{1,2, \ldots, Q-1\}$ so that each element of this subset is found to be put on at least one of the excited sites of all the polymers the cluster is composed of. Then we can assign to the cluster an integer $\tilde{q}$ so that $(\tilde{q}-1)$ is the number of the elements of the subset. We can prove [3] that the Taylor expansion of the $W\left(l_{x} \times l_{y} ; q\right)$ with respect to $z$ includes the contribution from all the clusters of polymers in the standard cluster expansion that have $\tilde{q}=q$ and that can be embedded into the $l_{x} \times l_{y}$ lattice but cannot be embedded into any of its rectangular sub-lattices.

So the series expansion of the $W\left(l_{x} \times l_{y} ; q\right)$ starts from the order $z^{n\left(l_{x}, l_{y}, q\right)}$ with $n\left(l_{x}, l_{y}, q\right)$ given in the following. It is enough to consider only for $l_{x} \leq$ $l_{y}$. We first define $k \equiv\left[(q-1) /\left(l_{x} l_{y}\right)\right]$, where we denote $[p]$ as the maximum integer that is less than $p$. In the case of $k=0$ ( namely $q-1 \leq l_{x} l_{y}$ ), the cluster that contributes to the lowest-order term of the expansion series of the $W\left(l_{x} \times l_{y} ; q\right)$ consists of a single polymer. We know in this case that

$$
n\left(l_{x}, l_{y}, q\right)=\left\{\begin{array}{cll}
2\left(l_{x}+l_{y}\right)+q-2 & \text { for } & q \leq l_{x}+l_{y} \\
l_{x}+l_{y}+2 q-2 & \text { for } & q>l_{x}+l_{y} .
\end{array}\right.
$$

Examples of the clusters that correspond to these two cases in Eqs. (12) and (13) are presented in Fig.1 (a) and (b), respectively. In the figures the integers denote the excited spin variables at the sites and the solid lines represent the excited bonds. In the case of $k \geq 1$ ( namely $q-1>l_{x} l_{y}$ ), the cluster that contributes to the lowest order term is composed of $k+1$ 
polymers, among which a polymer consists of $q^{\prime} \equiv q-1-k l_{x} l_{y}$ sites and each of the other $k$ polymers consists of the $l_{x} \times l_{y}$ sites. Then if $q^{\prime} \leq l_{x}^{2}$

$$
n\left(l_{x}, l_{y}, q\right)=\left\{\begin{array}{c}
k\left\{l_{x}\left(l_{y}+1\right)+\left(l_{x}+1\right) l_{y}\right\}+2 q^{\prime}+2 i+1 \\
\text { for } \quad q^{\prime} \leq i(i+1) \\
k\left\{l_{x}\left(l_{y}+1\right)+\left(l_{x}+1\right) l_{y}\right\}+2 q^{\prime}+2 i+2 \\
\text { for } \quad q^{\prime}>i(i+1)
\end{array}\right.
$$

where $i \equiv\left[\sqrt{q^{\prime}}\right]$. Examples of the clusters that correspond to these two cases in Eqs. (14) and (15) are presented in Fig.2 (a) and (b), respectively. If $q^{\prime}>l_{x}^{2}$

$$
n\left(l_{x}, l_{y}, q\right)=k\left\{l_{x}\left(l_{y}+1\right)+\left(l_{x}+1\right) l_{y}\right\}+2 q^{\prime}+2 l_{x}+j+1
$$

where $j \equiv\left[\left(q^{\prime}-l_{x}^{2}\right) / l_{x}\right]$. Example of the cluster that corresponds to Eq. (16) is presented in Fig.2 (c). To obtain the expansion series to order $z^{N}$, we should take into account all the combinations of $l_{x}, l_{y}$ and $q$ that satisfy $n\left(l_{x}, l_{y}, q\right) \leq N$ in the summation of Eq. (7) and should evaluate each of the $W$ 's to the order of $z^{N}$.

We should stress that the improved algorithm described above enables us to calculate the series for large $Q$ that is substantially longer than the case when one would apply the standard finite-lattice method naively [9, 11]. In the latter case one would define

$$
\tilde{H}\left(\Lambda ; \xi_{Q}\right)=-\ln \left[Z\left(\Lambda ; \xi_{Q}\right)\right]
$$

and

$$
\tilde{W}\left(\Lambda ; \xi_{Q}\right)=\tilde{H}\left(\Lambda ; \xi_{Q}\right)-\sum_{\Lambda^{\prime} \subset \Lambda} \tilde{W}\left(\Lambda^{\prime} ; \xi_{Q}\right)
$$

for the rectangular $l_{x} \times l_{y}$ sub-lattice $\Lambda$. Here the variable $s_{i}$ takes all integer values $0 \leq s_{i} \leq Q-1$ for each site $i \in \Lambda$ in the calculation of the partition function $Z\left(\Lambda ; \xi_{Q}\right)$. Then the free energy density in the infinite-volume limit would be given by

$$
f=\sum_{l_{x}, l_{y}} \tilde{W}\left(l_{x} \times l_{y} ; \xi_{Q}\right) .
$$

The series expansion of this $\tilde{W}\left(l_{x} \times l_{y} ; \xi_{Q}\right)$ starts from the order of $z^{2\left(l_{x}+l_{y}\right)}$. The clusters that give the contribution to this order are the clusters consisting of a single polymer, an example of which is depicted in Fig.3. So for the 
series expansion to order $z^{N}$, one should take into account all the rectangular lattices that satisfy $2\left(l_{x}+l_{y}\right) \leq N$ in the summation of Eq. (19). The improved algorithm might appear to be more complicated than this standard algorithm, since the former involves the summation with respect to $q$ as well as the summation with respect to $l_{x} \times l_{y}$. The CPU time and memory for evaluating the partition functions of the finite-size lattices needed to obtain the series to the same order are, however, much smaller in the improved algorithm. In order to obtain the series to $z^{41}$, for instance, we should calculate the partition functions up to the lattice-size of $10 \times 10$ either in the standard algorithm or in the improved algorithm. In the standard algorithm, the partition function with $q=Q$ is needed for this maximum size of the lattice. On the other hand, in the improved algorithm the partition function with $q=2$ is enough for this size of the lattice.

\section{Series}

Using the improved algorithm of the finite-lattice method described in the previous section we have calculated the low-temperature series for the free energy density $f$, magnetization $M$ and susceptibility $\chi$ in the zero field for the Potts model on the square lattice to order $z^{41}$ for $Q=5-50$. The obtained coefficients are listed in Table 1 and 2 only for $Q=20$ and 50, where we give the series for $z=\exp (f)$ instead of the free energy density $f$, as well as the series for the magnetization and susceptibility. If the reader would like to know the coefficients for the other values of $Q$, he can get them from the authors by e-mail or from the authors' web-site. 10 The calculation was performed by FACOM-VPP500 at KEK (Tsukuba) and FACOM-VP2600 at Kyoto University Data Processing Center. The maximum size of the used main memory was about 500Mbytes. We have checked that each of the $W\left(l_{x} \times l_{y} ; q\right)$ 's in eq. (7) starts from the correct order of $z^{n\left(l_{x}, l_{y}, q\right)}$ described in the previous section.

The series were obtained previously using the standard finite-lattice method by Bhanot et al [9] to order $z^{25}$ for $Q=8$ and by Briggs et al [11] to order $z^{39}, z^{39}, z^{39}, z^{35}, z^{31}$ and $z^{31}$ for $Q=5,6,7,8,9$ and 10 , respectively. The series were also calculated by Kihara et al [12] to order $z^{16}$ for general $Q$ and zero field and by Straley and Fisher 13] for general $Q$ and general field to order $z^{13}$ using the graphical method. The series coefficients obtained here by us are consistent with the coefficients obtained by these people to their order. 
We note that in the standard finite-lattice method the maximum order of the obtainable expansion series reduces when one increases the value of the $Q$, while the improved algorithm enables us to calculate the series to the same order for all the $Q$ that are larger than or equal to some value. In fact, we can calculate the series to the order of $u^{41}$ for an arbitrarily large $Q(>17)$ using the $W\left(l_{x}, l_{y} ; q\right)$ 's that have been evaluated here to obtain the series to order $u^{41}$ for $Q=17$, although we have stopped it at $Q=50$. This can be understood by noticing from Eqs. (12)-(16) that the series expansion of the $W\left(l_{x}, l_{y} ; q\right)$ for arbitrary $l_{x}, l_{y}$ and $q$ with $q>17$ starts from the order that is higher than $z^{41}$ and does not contribute to the expansion series to the order of $z^{41}$.

\section{Analysis of the series}

The $Q$-state Potts model with $Q>4$ has a first-order phase transition. For the first-order phase transition, some important quantities, such as the susceptibility and specific heat, have finite values at the critical point, in contrast with the fact that these values are infinite for the second-order phase transition.

The infiniteness of the critical value for the second-order phase transition sometimes makes the analysis easier; if the physical quantity would have a strong singularity, we would be able to determine the critical exponent with a satisfactory accuracy by the differential approximations such as the Padé analysis.

On the other hand, in the case of the first-order phase transition, we have to integrate the differential approximants to determine the critical amplitude. This integration, however, has a subtle problem discussed below, and as a result, we must choose the approximants carefully.

In analyzing the expansion series, we use homogeneous or inhomogeneous first order differential approximants [17], in which the approximants to a function $f(z)$ satisfy

$$
Q_{1}(z) f^{\prime}(z)+Q_{2}(z) f(z)=R(z) .
$$

Here, $Q_{1}(z), Q_{2}(z)$ and $R(z)$ are polynomials of order $M_{1}, M_{2}$, and $M$, respectively, which are determined so that Eq. (20) is satisfied to the order of $z^{N}$ with $N$ the maximum order of the expansion series of the $f(z)$. If $R(z)$ is identically zero, the approximant is just the D-log Padé approximant. 
To obtain the value of $f(z)$ at the critical point $z=z_{c}$, we must integrate the function $f^{\prime}(z)=\left\{-Q_{2}(z) f(z)+R(z)\right\} / Q_{1}(z)$ from 0 to $z_{c}$. For the first order phase transition, the denominator $Q_{1}(z)$ of $f^{\prime}(z)$ has in most cases a zero close to the critical point. The zero point is usually slightly above the critical point on the real axis of $z$. It is sometimes, however, below the critical point, then the integral of $f^{\prime}(z)$ will diverge and we should abandon such an approximant. Even if the zero point is above the critical point, it is so close to the critical point that its subtle fluctuation makes the fluctuation of the critical value $f\left(z_{c}\right)$ rather large.

At first, in order to examine the accuracy of the analysis of the series described above, we apply it to the free energy density $f_{c}$ at the critical point $\left(z_{c}=1 /(1+\sqrt{Q})\right)$, the latent heat $\Delta U$ and the magnetization gap $\Delta M$, whose exact values were obtained by Baxter 8 . In Table 3 the estimates of the free energy density, latent heat and magnetization gap from the longest series $(N=41)$ are presented for $Q \geq 7$, and their exact values are in parentheses. These estimates obtained by the analysis of the series may contain errors by two reasons; One of them is the statistical fluctuation among the approximants with the different orders $M_{1}, M_{2}$ and $M$ of the polynomials $Q_{1}(z)$ and $Q_{2}(z)$ in Eq. (20) but with the same order $N$ of the original series. About these statistical errors we find two common tendency; i) If the order $M$ is too large, the convergence of the approximants is not so good, and if the orders $M_{1}$ and $M_{2}$ are too small, the integral of $f^{\prime}(z)$ diverges frequently, therefore we restrict the order $M$ to be $M=-1$ (D-log Padé approximant) or $M=0-6$ (inhomogeneous differential approximants) and the orders $M_{1}$ and $M_{2}$ to satisfy $\left|M_{1}-M_{2}\right| \leq 10$. ii) For smaller $Q$, some approximants are finite at the critical point but apparently far from the exact value, then we must exclude them. We present only the statistical errors in Table 3. The other error is from the finiteness of the series. If there is discrepancy between an estimate for the finite series and the corresponding exact value beyond the statistical error for each $N$, we may say that the discrepancy is caused by the finiteness of the series and the error arising from the finiteness of the series has the order of magnitude of this discrepancy.

Now, we present the result of the individual analysis for the free energy density, the latent heat and the magnetization gap.

For the free energy density $f_{c}$, both the inhomogeneous and homogeneous differential approximants give well-converged results, the latter converging more excellently. The data in Table 3 are from the latter. For $Q \gtrsim 30$, 
the estimates from the D-log Padé approximants agree with the exact value within one standard deviation. On the other hand, for $Q \lesssim 20$, the estimates are larger than the exact value, and do not agree with it within one standard deviation. As an example in Fig.4, we present a plot of the estimates for $Q=20$ versus the number $N$ of terms of the truncated series. The difference between the estimates and the exact value is about 0.01 percent while the fluctuation of the approximants is about 0.005 percent, the former is about 2 times larger than the latter. The ratio of the difference between the estimate and the exact value to the statistical fluctuation becomes larger for smaller $Q$. Nevertheless, the figure also exhibits the tendency that the difference between the estimates and the exact value decreases with the increase of the number of terms. (We should mention that the errorbars for $35 \leq N \leq 38$ are relatively small. It is because, for these $N$, some approximants have divergent integral of $f^{\prime}(z)$ and the remaining fewer approximants happen to be very close.) Therefore, this difference can be expected to shrink for longer series. It would, however, be difficult to extrapolate the data to $N \rightarrow \infty$ to estimate the exact value, if it were not known, since we do not know what asymptotic behavior the approximants should follow with respect to $N$.

For the latent heat, the inhomogeneous differential approximants give more convergent results than the D-log Padé approximants, in contrast with the case of the free energy density. Then, by using the inhomogeneous differential approximants, we can obtain about 60 estimates of the latent heat for each model with $Q \geq 7$. For $Q \gtrsim 40$, the estimates coincide with the exact value within one standard deviation. For $Q \lesssim 30$, although the estimates do not agree with the exact value within one standard deviation, we find that their difference becomes smaller for larger number of terms of the expansion series and so we can say that the difference would disappear for long enough series, as in the previous case of the free energy density.

For the magnetization gap, about 10 estimates obtained by the D-log Padé approximants converge well for each $Q$, while those obtained by the inhomogeneous differential approximants do not. Although it does not agree with the exact value within one standard deviation except for $Q \simeq 50$, the estimates themselves are very close to the exact value; for example, in the case of $Q=20$, the difference between the estimates and the exact value is about 0.015 percent. Further Fig. 5 shows that the differences would disappear for larger number of terms as well as in the case of the free energy density and the latent heat.

Now using the same method as the above, we estimate the values of the 
susceptibility and specific heat at the critical point, whose exact values are not known.

The behavior of the differential approximants for the susceptibility is like that for the magnetization gap. The D-log Padé approximants for the susceptibility show better convergence than the inhomogeneous approximants, although the errors of the estimates for the susceptibility are larger than those for the magnetization gap. The estimates from the longest series $(N=41)$ are listed in Table 4 , and our estimates for $7 \leq Q \leq 10$ are consistent with the estimates obtained by Briggs et al[11]. For $Q=10,15$, 20, Monte Carlo estimates are given by Janke et al 23]. As their definition of the susceptibility is different from ours in the normalization factor, the results in Ref 23] should multiplied by a factor of $\{q /(q-1)\}^{2}$ to fit into our definition. The estimates obtained from Ref [23] are $3.777 \pm 0.035(Q=10)$, $0.7052 \pm 0.0033(Q=15)$ and $0.30242 \pm 0.00050(Q=20)$. Our corresponding estimates are $2.78 \pm 0.14(Q=10), 0.657 \pm 0.017(Q=15)$ and $0.2938 \pm 0.0036(Q=20)$. Although there are differences over one standard deviation, which are especially large for $Q=10$, we may expect that the differences would disappear for larger $Q$.

For the specific heat, the estimates obtained by the D-log Padé approximants give well converging results, while the inhomogeneous differential approximants do not. We list the estimates from the longest series $(N=41)$ by the D-log Padé approximants in Table 5. The errors of the estimates for the specific heat are smaller than those for the susceptibility. Nevertheless, our estimates for $7 \leq Q \leq 10$ are inconsistent with the estimates obtained by Briggs et al[11]. Therefore we should check the validity of our approximants in some way.

Fortunately, by the duality relation [8], we can obtain the exact value of the gap of the specific heat as follows [8];

$$
C_{v}^{+}-C_{v}^{-}=\frac{\beta^{2} \Delta U}{\sqrt{Q}}
$$

In Table 5, we also list the estimates of the gap of the specific heat, where the specific heat $C_{v}^{+}$is calculated using the high-temperature expansion series obtained from the low-temperature series by the duality. It is clear that for $Q \geq 11$ there exists the gap of the specific heat and more over almost all the estimated values of the gap of the specific heat coincide with the exact values given by Eq. (21) within the standard deviation. Therefore, we convince the validity of our estimates for the specific heat. 
Finally we compare our estimates of the specific heat at the critical point with the estimates obtained from the large- $Q$ expansion series that was recently obtained by Bhattacharya et al [18], and with the ones obtained from Monte Carlo simulations [19, 20, 21, 22, 23]. In Table 6, we show our estimates of the specific heat again and the estimates obtained from the large- $Q$ expansion series and the Monte Carlo simulations. For $Q=$ $7,8,10,15,20,30$, the values of the estimates from the large- $Q$ expansion are sited in Ref [18], the others are calculated by the regularized logarithmic Padé approximants given in Ref 18]. Among the Monte Carlo results those of Ref. 23] have small statistical errors and they are consistent with the results of the large- $Q$ expansion for $Q=10,15,20$. Our estimates from the low-temperature series are systematically smaller than the estimates from the large- $Q$ expansion. In Fig.6 and 7, we present a plot of the estimates of the specific heat $C_{v}^{-}$for $Q=20$ and $Q=50$, respectively, versus the number $N$ of the terms of the series truncated. We can see that the estimates are approaching the result of the large- $Q$ expansion from below for each $Q$ and that they approach faster for larger $Q$. Thus we can expect that, if we extrapolate the estimates in some way to $N \rightarrow \infty$ for each $Q$, we would obtain the estimates that may be more consistent with the result of the large- $Q$ expansion, although we do not know how to make the extrapolation correctly, as mentioned above.

\section{Summary}

We obtained the low-temperature series for the free energy, magnetization and susceptibility of the Potts model on the square lattice.

Using an improved algorithm of the finite lattice method, we extended the series from those given by the standard algorithm of the finite lattice method. Our improved algorithm is more efficient for the higher-state Potts models.

Using the new series, we calculated the critical values of the free energy density, latent heat, magnetization gap, susceptibility and specific heat.

The estimated values of the former three are very close to the known exact values. The values of the latter two, which are not known exactly, are obtained for various $Q$ with high precision. Especially, the estimates of the specific heat are very close to the estimates from the large $Q$ expansion series. 


\section{References}

[1] T. de Neef and I. G. Enting, J. Phys. A10 (1977) 801;

I. G. Enting, J. Phys. A11 (1978) 563; Aust. J. Phys. 31 (1978) 515.

[2] M. Creutz, Phys. Rev. B43 (1991) 10659.

[3] H. Arisue and T. Fujiwara, Prog. Theor. Phys. 72 (1984) 1176;

Preprint RIFP-588 (1985 unpublished);

H. Arisue, Nucl. Phys. B (Proc. Suppl.) 34 (1994) 240.

[4] I. G. Enting, J. Phys. A13 (1980) 3713;

G. Bhanot, J. Stat. Phys. 60 (1990) 55.

[5] H. Arisue, Nulc. Phys. B446[FS] (1995) 373.

[6] M. Hasenbusch and K. Pinn, Physica A203 (1994) 189.

[7] R. B. Potts, Proc. Camb. Phil. Soc. 48 (1952) 106.

[8] R. J. Baxter, J. Phys. C6 (1973) L445; J. Phys. A15 (1982) 3329.

[9] G. Bhanot, M. Creutz, U. Glässner, I. Horvath , J. Lacki, K. Schilling and J. Weckel, Phys. Rev. B48 (1993) 6183.

[10] E-mail address: arisue@ipc.osaka-pct.ac.jp and

URL of the web-site: http://www2.yukawa.kyoto-u.ac.jp/ ${ }^{\sim}$ arisue/

[11] K. M. Briggs, I. G. Enting and A. J. Guttmann, J. Phys. A27 (1994) 1503.

[12] T. Kihara, Y. Midzuno and T. Shizume, J. Phys. Soc. Jap. 9 (1954) 681.

[13] J. P. Straley and M. E. Fisher, J. Phys. A6 (1973) 1310.

[14] A. J. Guttmann and I. G. Enting, J. Phys. A26 (1993) 807.

[15] C. Domb, Phase Transitions and Critical Phenomena vol.3 ed. C. Domb and M. S. Green (Academic, New York 1974).

[16] G. Münster, Nucl. Phys. B180[FS2] (1981) 23. 
[17] A. J. Guttmann and G. S. Joyce, J. Phys. A5 (1972) L81; Padé approximants and their applications, eds. P. Graves-Morris (Academic Press, New York, 1973);

G. L. Hunter and G. A. Baker, Phys. Rev, B19 (1979) 3808;

M. E. Fisher and H. Au-Yang, J. Phys. A12 (1979) 1677.

[18] T. Bhattacharya, R. Lacaze and A. Morel, Preprint SPhT-96/001 (1996).

[19] A. Billoire, R. Lacaze and A. Morel, Nucl. Phys. B370 (1992) 773.

[20] A. Billoire, T. Neuhaus and B. Berg, Nucl. Phys. B396 (1993) 779.

[21] W. Janke, B. A. Berg and M. Katoot, Nucl.Phys. B382 (1992) 649.

[22] K. Rummukainen, Nucl. Phys. B390 (1993) 621.

[23] W. Janke and S. Kappler, Europhys. Lett. 31 (1995) 345. 
Table 1: The low-temperature expansion coefficients for the free energy, magnetization and susceptibility for the $Q=20$ square lattice Potts model.

\begin{tabular}{|c|c|c|c|}
\hline$n$ & $\bar{z}$ & $\bar{M}$ & $\bar{\chi}$ \\
\hline 0 & 1 & $\overline{1}$ & $\overline{0}$ \\
\hline 1 & 0 & 0 & 0 \\
\hline 2 & 0 & 0 & 0 \\
\hline 3 & 0 & 0 & 0 \\
\hline 4 & 19 & -20 & 19 \\
\hline 5 & 0 & 0 & 0 \\
\hline 6 & 38 & -80 & 152 \\
\hline 7 & 684 & -1440 & 2736 \\
\hline 8 & -589 & 1460 & -2280 \\
\hline 9 & 4104 & -12960 & 36936 \\
\hline 10 & 34466 & -109520 & 323912 \\
\hline 11 & -46512 & 138240 & -199728 \\
\hline 12 & 483398 & -2040420 & 7678261 \\
\hline 13 & 1614240 & -7086240 & 30536496 \\
\hline 14 & -2010808 & 4671200 & 19059128 \\
\hline 15 & 48638556 & -259282080 & 1242232920 \\
\hline 16 & 66750002 & -417704060 & 2800285550 \\
\hline 17 & 94851648 & -1155653280 & 11160075600 \\
\hline 18 & 4264583402 & -27837269920 & 166147691080 \\
\hline 19 & 2711283660 & -28967068800 & 314891078616 \\
\hline 20 & 36660260676 & -326575493500 & 2700423490565 \\
\hline 21 & 350530337736 & -2773117509120 & 20580457476816 \\
\hline 22 & 226902725452 & -3504413044080 & 47276982916744 \\
\hline 23 & 5653101156048 & -54127038428640 & 481746412796832 \\
\hline 24 & 28469510466547 & -273739383621540 & 2536124771313858 \\
\hline 25 & 42704598074544 & -626534197990560 & 8388189200399472 \\
\hline 26 & 700159265957050 & -7500005076811440 & 75083308577493088 \\
\hline 27 & 2423368661949468 & -28449325073826720 & 325937254907403792 \\
\hline 28 & 7731033540174246 & -110572911429835580 & 1480698106281593115 \\
\hline 29 & 78953345947533348 & -956758345892651520 & 10938227296679669016 \\
\hline 30 & 233171802367220678 & -3290695663943877920 & 45003875885372649120 \\
\hline 31 & 1207925854600942620 & -17857155839057961120 & 248795624942616383064 \\
\hline 32 & 8578891752427054593 & -118198071379878114580 & 1550417616117914081584 \\
\hline 33 & 26279052771743353332 & -427018427440815394080 & 6633731834136735494256 \\
\hline 34 & 169448419303276320478 & -2669246990084147723840 & 39743309408406296543112 \\
\hline 35 & 934014456459287376408 & -14635750107536528768640 & 219466703191297256269272 \\
\hline 36 & 3342530604057094050901 & -59864493412976212314860 & 1016877625830611545531997 \\
\hline 37 & 22227466452797269128528 & -378821839108831184988000 & 6119224442354906298876816 \\
\hline 38 & 105216422923458425776276 & -1862780050724742025689040 & 31557668972616720906032400 \\
\hline 39 & 449173545929305443593316 & -8659232479851255144790560 & 157936029967724328819066816 \\
\hline 40 & 2809270008061904464069897 & -52184954811154800744150940 & 921011351332383770376427924 \\
\hline 41 & 12477877823064242776774212 & -246220110417995423090111520 & 4633614244759932587057525712 \\
\hline
\end{tabular}


Table 2: The low-temperature expansion coefficients for the free energy, magnetization and susceptibility for the $Q=50$ square lattice Potts model.

\begin{tabular}{|c|c|c|c|}
\hline$n$ & $\bar{z}$ & $\bar{M}$ & $\bar{x}$ \\
\hline 0 & 1 & 1 & $\overline{0}$ \\
\hline 2 & 0 & 0 & 0 \\
\hline 3 & 0 & 0 & 0 \\
\hline 5 & 0 & 0 & 0 \\
\hline 6 & 98 & -200 & 392 \\
\hline 7 & 4704 & -9600 & 18816 \\
\hline 8 & -4459 & 11150 & -20580 \\
\hline 11 & -1025472 & 3225600 & -7018368 \\
\hline 12 & 12281948 & -50077050 & 193485271 \\
\hline 13 & 89987520 & -370953600 & 1510567296 \\
\hline 14 & -162228808 & 567974000 & -886343752 \\
\hline 15 & 3720812256 & -19045411200 & 92683335360 \\
\hline 16 & 12381176822 & -67093148150 & 376836950000 \\
\hline 17 & 4469044608 & -89631139200 & 1066326898560 \\
\hline 23 & 5667428458933248 & -51068413852233600 & 452593396732200192 \\
\hline 24 & 49346646734282527 & -435539546923202850 & 3808046989807658388 \\
\hline 25 & 172491504405475584 & -1825893233439542400 & 19671214965561745152 \\
\hline 26 & 1938967535168345710 & -19591446789689634600 & 194639282941507768168 \\
\hline 27 & 13089424692665334048 & -135834659906560636800 & 1410212431991986624512 \\
\hline 28 & 63921872954358377616 & -750734284293452018450 & 8836405252541575381155 \\
\hline 29 & 628985731889425518048 & -7147786492756957612800 & 80157906305935393997376 \\
\hline 30 & 3839485035589391850158 & -45982543354085211648800 & 551763520397297550084960 \\
\hline 31 & 23463259463706802592160 & -302788074318283787164800 & 3888163728504881482313664 \\
\hline 32 & 203694326578125205781553 & -2595462149212992934427950 & 32749085439406082880398944 \\
\hline 33 & 1225877434229949054953952 & -16606999340819591568259200 & 224791274766846749241616896 \\
\hline 34 & 8555010161977020587142598 & -120783311584253799401189600 & 1691607730655033745533673792 \\
\hline 35 & 66800901314888122476718848 & -948877081581250695129561600 & 13374510959732980701033577152 \\
\hline 36 & 417354805969242736779031351 & -6276444566058217793336109650 & 93992501752971816579399237857 \\
\hline
\end{tabular}


Table 3: Estimates of the free energy density $f_{c}$, the latent heat $\Delta U$ and the magnetization gap $\Delta M$.

\begin{tabular}{|c|c|c|c|}
\hline$Q$ & $f_{c}$ & $\Delta U$ & $\Delta M$ \\
\hline 7 & $\begin{array}{c}-0.0430048 \pm 0.0000024 \\
(-0.0431119)\end{array}$ & $\begin{array}{c}0.35720 \pm 0.00883 \\
(0.35328)\end{array}$ & $\begin{array}{l}0.7681 \pm 0.0060 \\
(0.749565)\end{array}$ \\
\hline 8 & $\begin{array}{c}-0.0392927 \pm 0.0000055 \\
(-0.0393533)\end{array}$ & $\begin{array}{c}0.49058 \pm 0.00193 \\
(0.48636)\end{array}$ & $\begin{array}{l}0.8080 \pm 0.0056 \\
\quad(0.799837)\end{array}$ \\
\hline 9 & $\begin{array}{c}-0.0360255 \pm 0.0000044 \\
(-0.0360805)\end{array}$ & $\begin{array}{c}0.60375 \pm 0.00219 \\
(0.59967)\end{array}$ & $\begin{array}{l}0.8394 \pm 0.0020 \\
\quad(0.833261)\end{array}$ \\
\hline 10 & $\begin{array}{c}-0.0332047 \pm 0.0000035 \\
(-0.0332365)\end{array}$ & $\begin{array}{c}0.69832 \pm 0.00182 \\
(0.69605)\end{array}$ & $\begin{array}{l}0.8605 \pm 0.0020 \\
\quad(0.857107)\end{array}$ \\
\hline 11 & $\begin{array}{c}-0.0307291 \pm 0.0000024 \\
(-0.0307586)\end{array}$ & $\begin{array}{c}0.78047 \pm 0.00057 \\
(0.77860)\end{array}$ & $\begin{array}{l}0.87746 \pm 0.00072 \\
(0.875107)\end{array}$ \\
\hline 12 & $\begin{array}{c}-0.0285722 \pm 0.0000025 \\
(-0.0285900)\end{array}$ & $\begin{array}{c}0.85122 \pm 0.00027 \\
(0.84994)\end{array}$ & $\begin{array}{c}0.89053 \pm 0.00047 \\
(0.888878)\end{array}$ \\
\hline 13 & $\begin{array}{c}-0.0266652 \pm 0.0000023 \\
(-0.0266817)\end{array}$ & $\begin{array}{c}0.91316 \pm 0.00029 \\
(0.91216)\end{array}$ & $\begin{array}{c}0.90114 \pm 0.00032 \\
(0.899999)\end{array}$ \\
\hline 14 & $\begin{array}{c}-0.0249831 \pm 0.0000021 \\
(-0.0249931)\end{array}$ & $\begin{array}{c}0.96756 \pm 0.00012 \\
(0.96689)\end{array}$ & $\begin{array}{l}0.90989 \pm 0.00025 \\
(0.909086)\end{array}$ \\
\hline 15 & $\begin{array}{c}-0.0234812 \pm 0.0000018 \\
(-0.0234908)\end{array}$ & $\begin{array}{c}1.015966 \pm 0.000090 \\
(1.015414)\end{array}$ & $\begin{array}{l}0.92094 \pm 0.00124 \\
(0.916664)\end{array}$ \\
\hline 16 & $\begin{array}{c}-0.0221416 \pm 0.0000018 \\
(-0.0221471)\end{array}$ & $\begin{array}{c}1.059128 \pm 0.000067 \\
(1.058749)\end{array}$ & $\begin{array}{c}0.92351 \pm 0.00018 \\
(0.923075)\end{array}$ \\
\hline 17 & $\begin{array}{c}-0.0209338 \pm 0.0000015 \\
(-0.0209393)\end{array}$ & $\begin{array}{c}1.098023 \pm 0.000054 \\
(1.097700)\end{array}$ & $\begin{array}{c}0.92888 \pm 0.000012 \\
(0.928570)\end{array}$ \\
\hline 18 & $\begin{array}{c}-0.0198439 \pm 0.0000013 \\
(-0.0198486)\end{array}$ & $\begin{array}{c}1.133140 \pm 0.000041 \\
(1.132916)\end{array}$ & $\begin{array}{c}0.93358 \pm 0.00012 \\
(0.933332)\end{array}$ \\
\hline 19 & $\begin{array}{c}-0.0188560 \pm 0.0000011 \\
(-0.0188594)\end{array}$ & $\begin{array}{c}1.165129 \pm 0.000038 \\
(1.164923)\end{array}$ & $\begin{array}{c}0.937683 \pm 0.000094 \\
(0.937499)\end{array}$ \\
\hline 20 & $\begin{array}{c}-0.0179567 \pm 0.0000010 \\
(-0.0179586)\end{array}$ & $\begin{array}{c}1.194298 \pm 0.000036 \\
(1.194155)\end{array}$ & $\begin{array}{c}0.941318 \pm 0.000008 \\
(0.941176)\end{array}$ \\
\hline 30 & $\begin{array}{c}-0.0120422 \pm 0.0000004 \\
(-0.0120425)\end{array}$ & $\begin{array}{c}1.390466 \pm 0.000011 \\
(1.390442)\end{array}$ & $\begin{array}{c}0.962987 \pm 0.000006 \\
(0.962963)\end{array}$ \\
\hline 40 & $\begin{array}{c}-0.0089727 \pm 0.0000002 \\
(-0.0089727)\end{array}$ & $\begin{array}{c}1.498232 \pm 0.000008 \\
(1.498224)\end{array}$ & $\begin{array}{c}0.972980 \pm 0.000004 \\
(0.972930)\end{array}$ \\
\hline 50 & $\begin{array}{c}-0.0071096 \pm 0.0000001 \\
(-0.0071096)\end{array}$ & $\begin{array}{c}1.567525 \pm 0.000003 \\
(1.567522)\end{array}$ & $\begin{array}{c}0.978726 \pm 0.000001 \\
(0.978723)\end{array}$ \\
\hline
\end{tabular}


Table 4: Estimates of the susceptibility $\chi_{c}$.

\begin{tabular}{cc}
\hline$Q$ & $\chi\left(z_{c}\right)$ \\
\hline 5 & $154 \pm 19$ \\
6 & $49.5 \pm 4.1$ \\
7 & $19.0 \pm 1.3$ \\
8 & $8.09 \pm 0.29$ \\
9 & $4.55 \pm 0.20$ \\
10 & $2.78 \pm 0.14$ \\
11 & $1.900 \pm 0.047$ \\
12 & $1.372 \pm 0.026$ \\
13 & $1.036 \pm 0.027$ \\
14 & $0.815 \pm 0.019$ \\
15 & $0.657 \pm 0.017$ \\
16 & $0.539 \pm 0.016$ \\
17 & $0.4490 \pm 0.0080$ \\
18 & $0.3844 \pm 0.0061$ \\
19 & $0.3347 \pm 0.0047$ \\
20 & $0.2938 \pm 0.0036$ \\
30 & $0.1195 \pm 0.0002$ \\
40 & $0.0697 \pm 0.0002$ \\
50 & $0.0478 \pm 0.00004$ \\
\hline
\end{tabular}


Table 5: Estimates of the specific heat.

\begin{tabular}{ccccc}
\hline$Q$ & $C_{v}^{-}$ & $C_{v}^{+}$ & $\Delta C_{v}$ (series) & $\Delta C_{v}$ (exact) \\
\hline 7 & $52.98 \pm 0.61$ & $68.01 \pm 0.47$ & $15.0 \pm 0.76$ & 0.2234 \\
8 & $28.47 \pm 0.47$ & $30.17 \pm 0.06$ & $1.70 \pm 0.48$ & 0.3099 \\
9 & $20.03 \pm 0.27$ & $20.87 \pm 0.05$ & $0.84 \pm 0.27$ & 0.3841 \\
10 & $15.579 \pm 0.083$ & $16.294 \pm 0.034$ & $0.715 \pm 0.090$ & 0.4476 \\
11 & $12.856 \pm 0.061$ & $13.493 \pm 0.034$ & $0.637 \pm 0.070$ & 0.5021 \\
12 & $10.985 \pm 0.050$ & $11.622 \pm 0.055$ & $0.636 \pm 0.074$ & 0.5492 \\
13 & $9.598 \pm 0.032$ & $10.258 \pm 0.062$ & $0.659 \pm 0.070$ & 0.5901 \\
14 & $8.548 \pm 0.027$ & $9.204 \pm 0.020$ & $0.656 \pm 0.034$ & 0.6260 \\
15 & $7.721 \pm 0.028$ & $8.388 \pm 0.004$ & $0.666 \pm 0.029$ & 0.6576 \\
16 & $7.044 \pm 0.011$ & $7.739 \pm 0.002$ & $0.695 \pm 0.011$ & 0.6856 \\
17 & $6.4908 \pm 0.0086$ & $7.2061 \pm 0.0041$ & $0.715 \pm 0.010$ & 0.7106 \\
18 & $6.0251 \pm 0.0074$ & $6.7637 \pm 0.0095$ & $0.739 \pm 0.012$ & 0.7330 \\
19 & $5.6317 \pm 0.0061$ & $6.3859 \pm 0.0098$ & $0.754 \pm 0.012$ & 0.7532 \\
20 & $5.2913 \pm 0.0053$ & $6.0645 \pm 0.0069$ & $0.773 \pm 0.009$ & 0.7714 \\
30 & $3.4012 \pm 0.0010$ & $4.2919 \pm 0.0051$ & $0.8906 \pm 0.0052$ & 0.8861 \\
40 & $2.5864 \pm 0.0006$ & $3.5303 \pm 0.0019$ & $0.9439 \pm 0.0020$ & 0.9393 \\
50 & $2.1218 \pm 0.0004$ & $3.0936 \pm 0.0007$ & $0.9717 \pm 0.0008$ & 0.9668 \\
\hline
\end{tabular}


Table 6: Estimates of the specific heat: low-temperature series vs. large $Q$ series and Monte Carlo simulations.

\begin{tabular}{|c|c|c|c|}
\hline$Q$ & low-temperature series & large $Q$ series & Monte Carlo simulations \\
\hline 7 & $52.98 \pm 0.61$ & $69.6 \pm 0.5$ & $\begin{array}{c}50 \pm 10[19] \\
47.5 \pm 2.4 \\
44.4 \pm 2.2\end{array}$ \\
\hline 8 & $28.47 \pm 0.47$ & $36.9 \pm 0.2$ & \\
\hline 9 & $20.03 \pm 0.27$ & $24.1 \pm 0.2$ & \\
\hline 10 & $15.579 \pm 0.083$ & $17.98 \pm 0.02$ & $\begin{aligned} 12.7 & \pm 0.3[19] \\
17.81 & \pm 0.10[23\end{aligned}$ \\
\hline 11 & $12.856 \pm 0.061$ & $14.24 \pm 0.05$ & \\
\hline 12 & $10.985 \pm 0.050$ & $11.84 \pm 0.02$ & \\
\hline 13 & $9.598 \pm 0.032$ & $10.17 \pm 0.01$ & \\
\hline 14 & $8.548 \pm 0.027$ & $8.939 \pm 0.009$ & \\
\hline 15 & $7.721 \pm 0.028$ & $7.999 \pm 0.003$ & $8.004 \pm 0.019$ \\
\hline 16 & $7.044 \pm 0.011$ & $7.248 \pm 0.004$ & \\
\hline 17 & $6.4908 \pm 0.0086$ & $6.643 \pm 0.003$ & \\
\hline 18 & $6.0251 \pm 0.0074$ & $6.142 \pm 0.002$ & \\
\hline 19 & $5.6317 \pm 0.0061$ & $5.720 \pm 0.001$ & \\
\hline 20 & $5.2913 \pm 0.0053$ & $5.3612 \pm 0.0004$ & $\begin{aligned} 5.2 & \pm 0.2[19 \\
5.3612 & \pm 0.0055\end{aligned}$ \\
\hline 30 & $3.4012 \pm 0.0010$ & $3.41294 \pm 0.00005$ & \\
\hline 40 & $2.5864 \pm 0.0006$ & $2.58986 \pm 0.00002$ & \\
\hline 50 & $2.1218 \pm 0.0004$ & $2.123198 \pm 0.000006$ & \\
\hline
\end{tabular}




\section{Figure Captions}

Fig. 1: Examples of the cluster consisting of a single polymer $(k=0)$ that contributes to the lowest-order term of the expansion series for (a) $W\left(l_{x}=4, l_{y}=5 ; q=4\right)$ and (b) $W\left(l_{x}=4, l_{y}=5 ; q=11\right)$.

Fig. 2: Examples of the cluster consisting of two polymers $(k=1)$ that contributes to the lowest-order term of the expansion series for (a) $W\left(l_{x}=3, l_{y}=4 ; q=18\right)$, (b) $W\left(l_{x}=3, l_{y}=4 ; q=21\right)$ and (c) $W\left(l_{x}=\right.$ $\left.3, l_{y}=4 ; q=24\right)$.

Fig. 3: Examples of the cluster consisting of a single polymer that contributes to the lowest-order term of the expansion series for $\tilde{W}\left(l_{x}=\right.$ $\left.4, l_{y}=5 ; \xi_{Q}\right)$.

Fig. 4: The estimates of the free energy density $f_{c}$ for $Q=20$ versus the number of terms of the series. The solid line shows the exact value.

Fig. 5: The estimates of the magnetization gap $\Delta M$ for $Q=20$ versus the number of terms of the series. The solid line shows the exact value.

Fig. 6: The estimates of the specific heat $C_{v}^{-}$for $Q=20$ versus the number of terms of the series. The solid line shows the estimates obtained from the large- $Q$ expansion series by using the regularized logarithmic Padé approximants.

Fig. 7: The estimates of the specific heat $C_{v}^{-}$for $Q=50$ versus the number of terms of the series. The solid line shows the estimates obtained from the large- $Q$ expansion series by using the regularized logarithmic Padé approximants. 
(a)

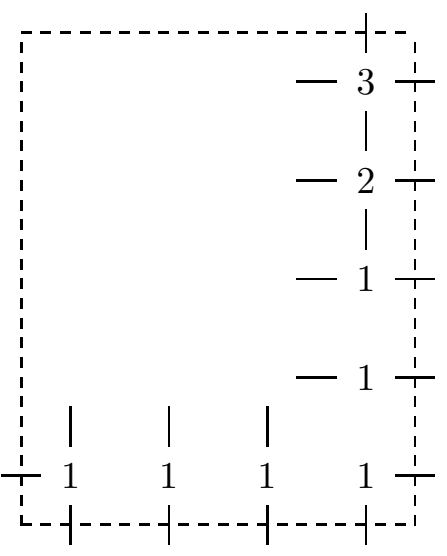

(b)

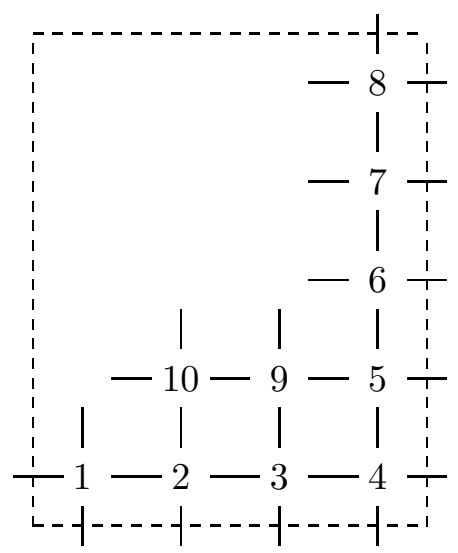

Figure 1: Examples of the cluster consisting of a single polymer $(k=0)$ that contributes to the lowest-order term of the expansion series for (a) $W\left(l_{x}=4, l_{y}=5 ; q=4\right)$ and (b) $W\left(l_{x}=4, l_{y}=5 ; q=11\right)$. 
(a)

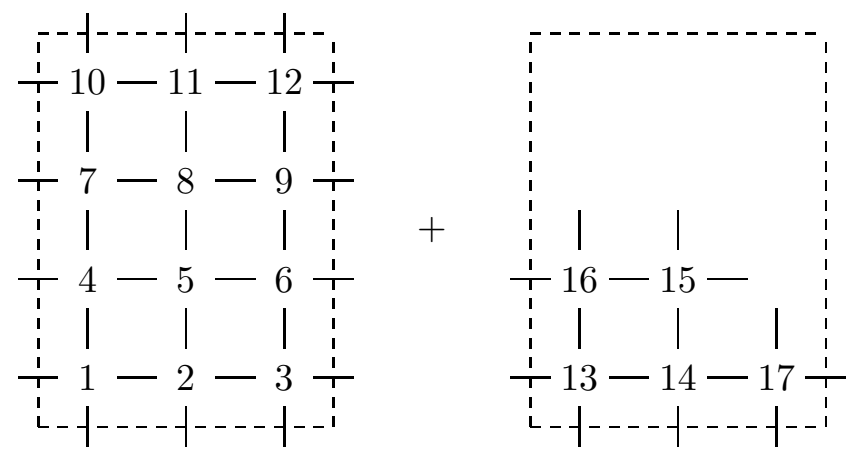

(b)

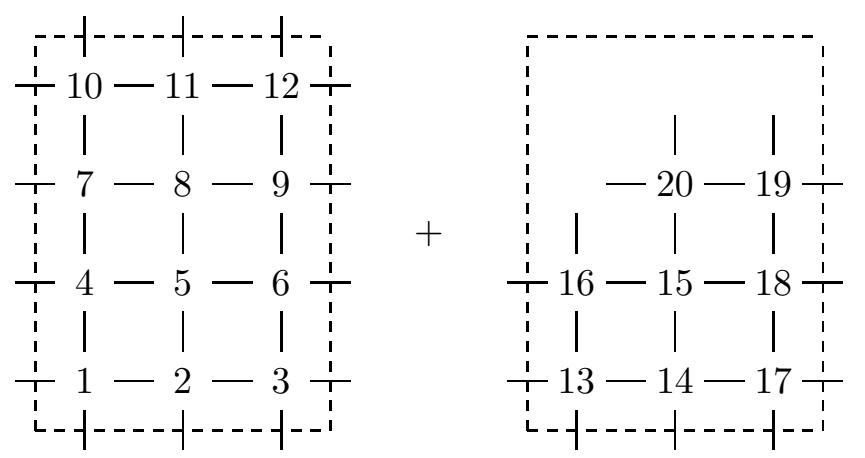

(c)
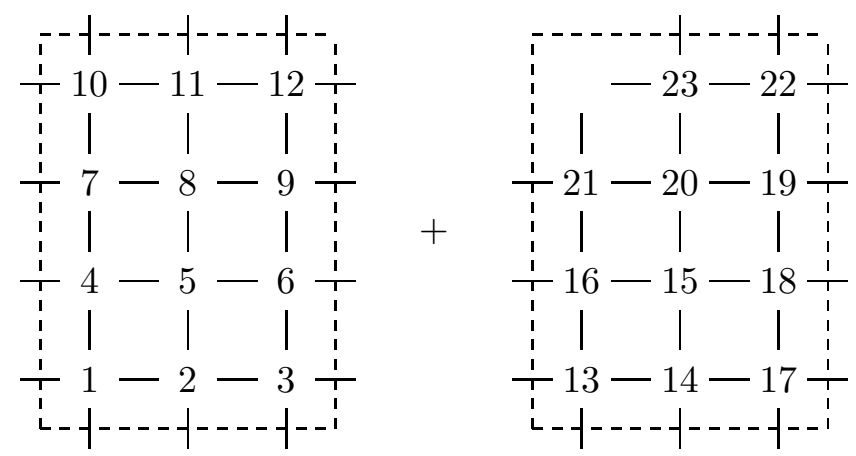

Figure 2: Examples of the cluster consisting of two polymers $(k=1)$ that contributes to the lowest-order term of the expansion series for (a) $W\left(l_{x}=3, l_{y}=4 ; q=18\right)$, (b) $W\left(l_{x}=3, l_{y}=4 ; q=21\right)$ and (c) $W\left(l_{x}=\right.$ $\left.3, l_{y}=4 ; q=24\right)$. 


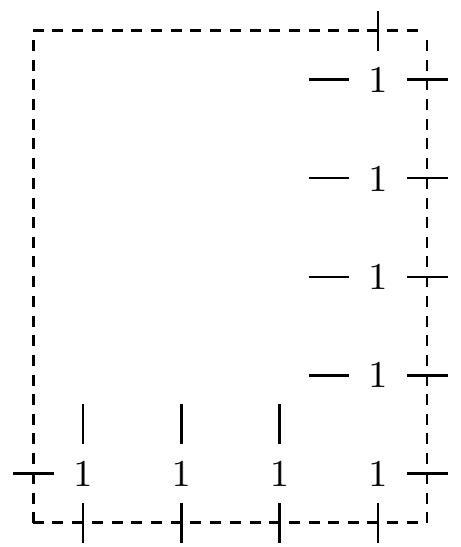

Figure 3: Examples of the cluster consisting of a single polymer that contributes to the lowest-order term of the expansion series for $\tilde{W}\left(l_{x}=\right.$ $\left.4, l_{y}=5 ; \xi_{Q}\right)$. 


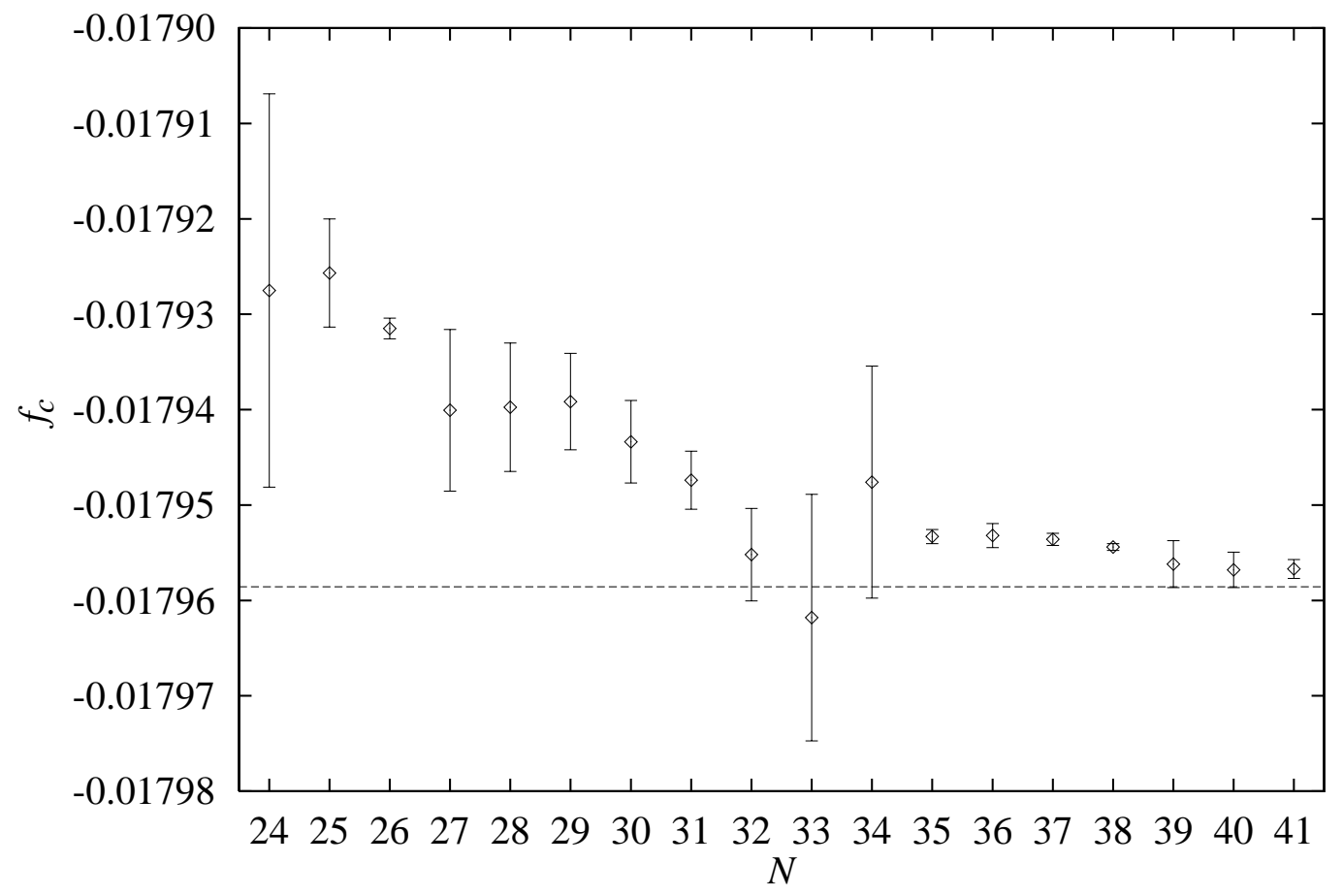

Figure 4: The estimates of the free energy density $f_{c}$ for $Q=20$ versus the number of terms of the series. The solid line shows the exact value. 


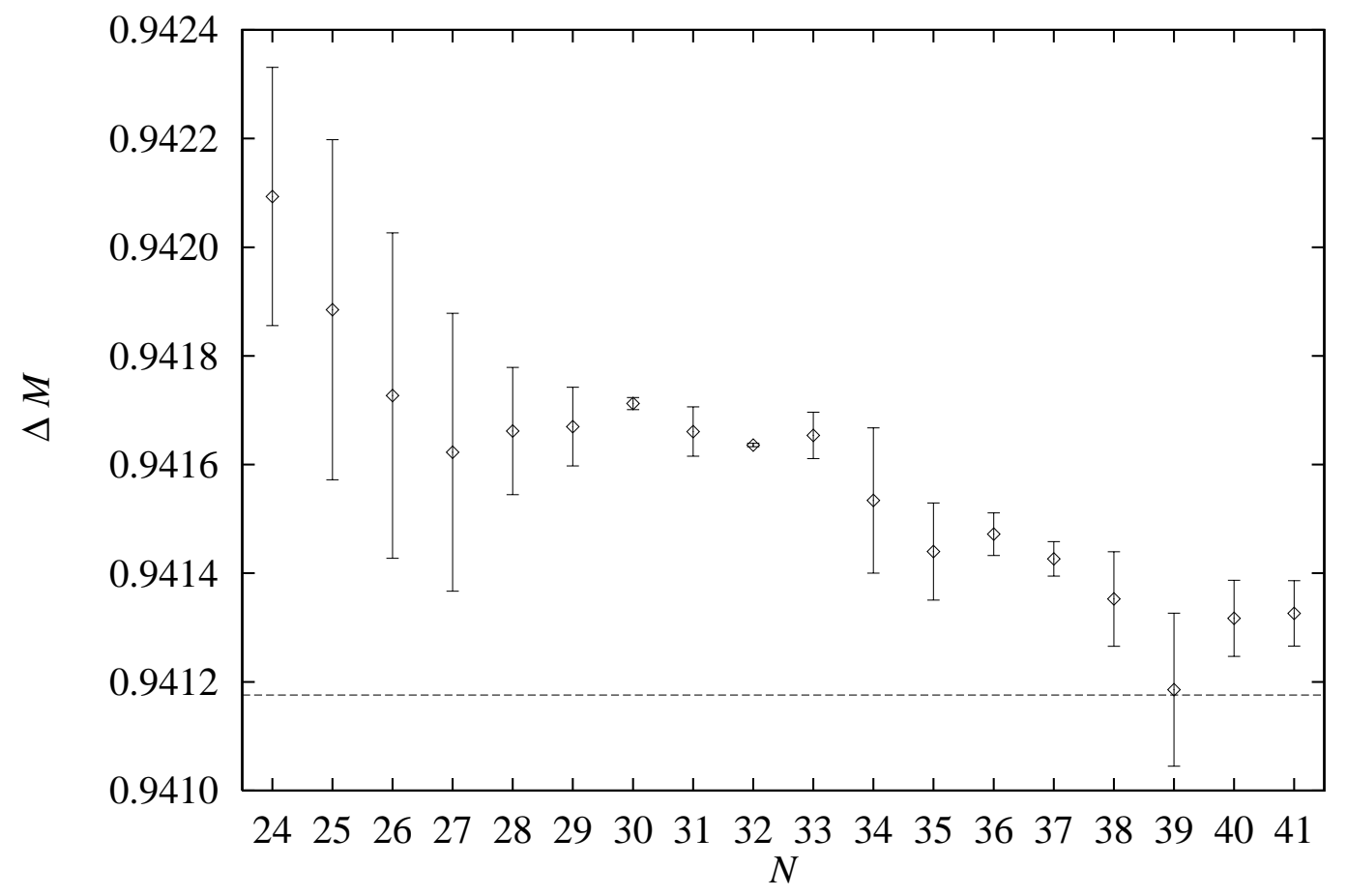

Figure 5: The estimates of the magnetization gap $\Delta M$ for $Q=20$ versus the number of terms of the series. The solid line shows the exact value. 


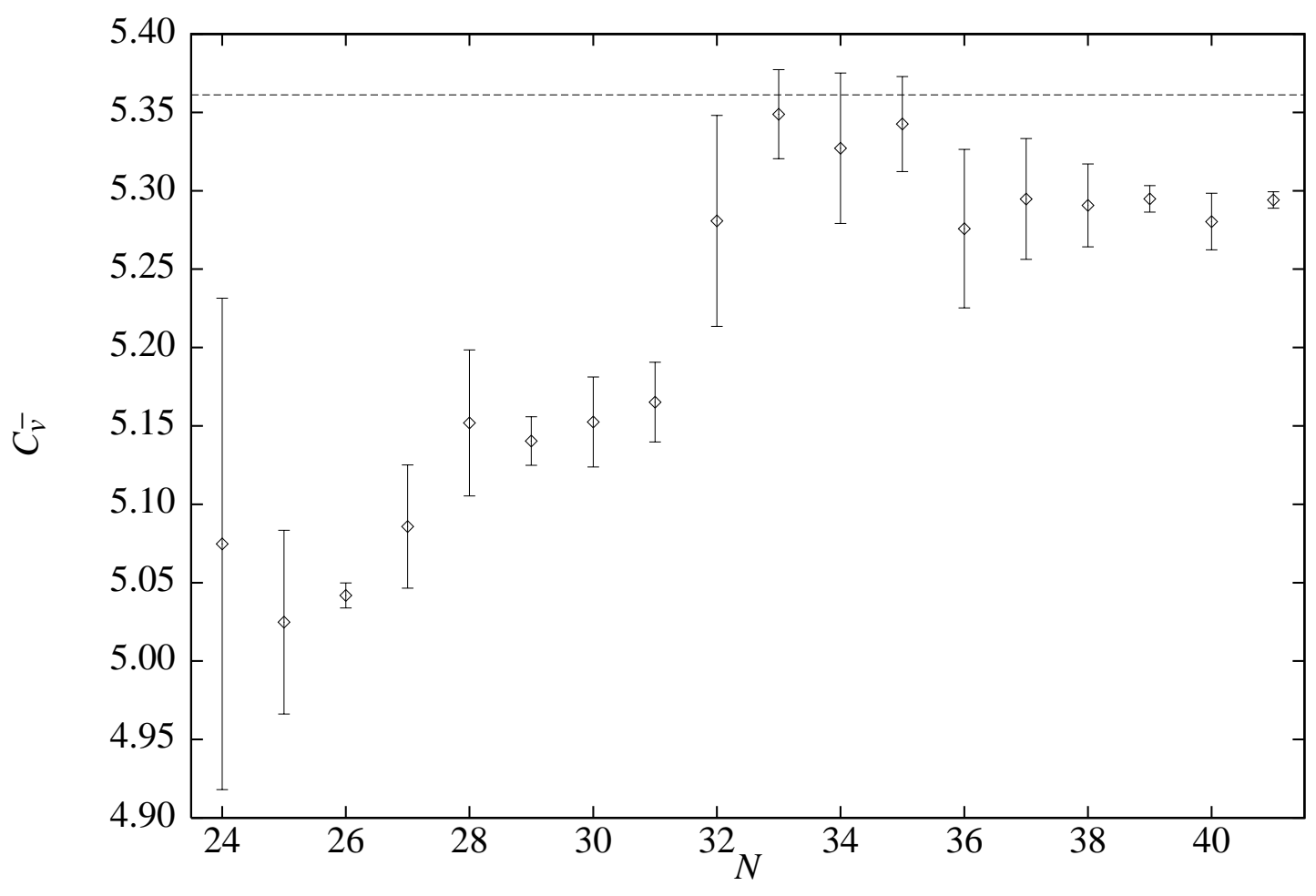

Figure 6: The estimates of the specific heat $C_{v}^{-}$for $Q=20$ versus the number of terms of the series. The solid line shows the estimates obtained from the large $Q$ expansion series by using the regularized logarithmic Padé approximants. 


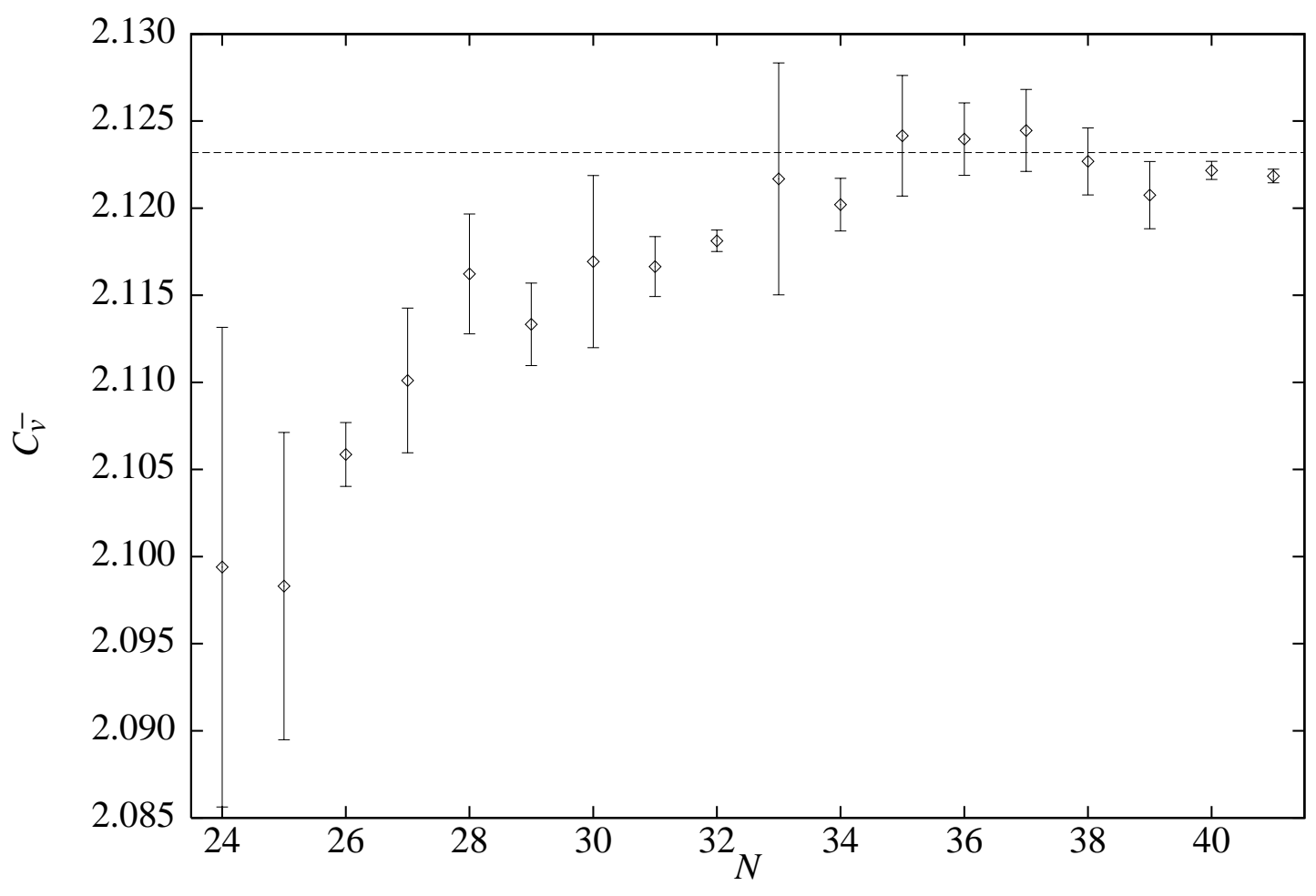

Figure 7: The estimates of the specific heat $C_{v}^{-}$for $Q=50$ versus the number of terms of the series. The solid line shows the estimates obtained from the large $Q$ expansion series by using the regularized logarithmic Padé approximants. 